\title{
FOXO mediates organismal hypoxia tolerance by regulating NF-KB in Drosophila
}

Elizabeth C Barretto ${ }^{1}$, Danielle M Polan ${ }^{1}$, Amy N Beever-Potts ${ }^{1}$, Byoungchun Lee ${ }^{1}$, Savraj S Grewal ${ }^{1 *}$

${ }^{1}$ Clark H Smith Brain Tumour Centre, Arnie Charbonneau Cancer Institute, Alberta Children's Hospital

Research Institute, and Department of Biochemistry and Molecular Biology Calgary, University of

Calgary, Alberta T2N 4N1, Canada.

*Author for correspondence (grewalss@ucalgary.ca)

KEY WORDS: hypoxia, Drosophila, FOXO, NF-KappaB, glucose metabolism, immunity 


\section{ABSTRACT}

Exposure of tissues and organs to low oxygen (hypoxia) occurs in both physiological and pathological conditions in animals. Under these conditions, organisms have to adapt their physiology to ensure proper functioning and survival. Here we define a role for the transcription factor FOXO as a mediator of hypoxia tolerance in Drosophila. We find that upon hypoxia exposure, FOXO transcriptional activity is rapidly induced in both larvae and adults. Moreover, we see that foxo mutant animals show

8 misregulated glucose metabolism in low oxygen and subsequently exhibit reduced hypoxia survival. We 9 identify the innate immune transcription factor, NF-KappaB/Relish, as a key FOXO target in the control 10 of hypoxia tolerance. We find that expression of Relish and its target genes are increase in a FOXO11 dependent manner in hypoxia, and that relish mutant animals show reduced survival in hypoxia.

12 Together, these data indicate that FOXO is a hypoxia inducible factor that mediates tolerance to low 13 oxygen by inducing immune-like responses. 


\section{INTRODUCTION}

Oxygen is essential for normal growth, development and functioning of tissues and organs. However, while the air we breathe contains $\sim 20 \%$ oxygen, even under healthy physiological conditions, our cells and tissues receive considerably lower levels. These can be anywhere from 1 to $10 \%$ oxygen depending on the tissue (McKeown, 2014). Hence, our tissues and organs need to function and maintain homeostasis at low levels of oxygen. This aspect of normal physiology is often neglected in tissue culture experiments where cells are routinely maintained in $20 \%$ oxygen. In addition, many diseases such as heart disease, stroke and chronic lung disease are characterized by severe oxygen deprivation (hypoxia) (Semenza, 2011). This hypoxia has deleterious effects on tissue metabolism and function, and can lead to death. Understanding how cells, tissues and organisms adapt to low oxygen is therefore an important question in biology.

One central hypoxic mechanism involves induction of the HIF-1 $\alpha$ transcription factor, which can control the expression of a diverse array of target genes that maintain cellular homeostasis in low oxygen (Semenza, 2014). The importance of HIF-1 $\alpha$ has been shown by loss of function genetic analysis in model organisms such as $C$ elegans, Drosophila and mice. For example, in C elegans and Drosophila, which are normally quite hypoxia-tolerant, HIF-1 $\alpha$ mutants die when exposed to low oxygen (Centanin et al., 2005; Jiang et al., 2001; Li et al., 2013). Tissue-specific mouse knockouts have also shown how HIF-1 $\alpha$ can control organ-level and whole-body adaptation to low oxygen in both physiological and pathological conditions (Boutin et al., 2008; Cramer et al., 2003; Huang et al., 2004; Mason et al., 2004; Schipani et al., 2001; Tomita et al., 2003). Compared to our understanding of HIF-1 $\alpha$, however, less is known about other transcription factors that are important in mediating hypoxia adaptation in animals.

The conserved transcription factor Forkhead Box-O (FOXO) is an important mediator of adaptation to stress in animals (Webb and Brunet, 2014). Studies in Drosophila have provided important insights into the role of FOXO as a regulator of organismal physiology. Here, different environmental stressors, such as starvation, oxidative stress, pathogens and ionizing radiation, have been shown to induce FOXO transcriptional activity (Borch Jensen et al., 2017; Dionne et al., 2006; Junger et al., 2003; Karpac et al., 2009; Karpac et al., 2011). Once induced, FOXO then directly controls the expression of an array metabolic and regulatory genes that together function to maintain organismal homeostasis and survival (Alic et al., 2011; Birnbaum et al., 2019; Gershman et al., 2007; Teleman et al., 2008). Indeed, genetic upregulation of FOXO is sufficient to promote stress resistance in Drosophila, and it is one of the most effective ways to extend lifespan (Alic et al., 2014; Demontis and Perrimon, 2010; Giannakou et al., 2004; Hwangbo et al., 2004; Kramer et al., 2008). 
In this paper, we report our work using Drosophila to explore hypoxia tolerance. In their natural ecology, Drosophila grow in rotting, fermenting food rich in microorganisms - an environment likely characterized by low ambient oxygen (Callier et al., 2015; Harrison et al., 2018; Markow, 2015). Probably as a consequence of this, they have evolved mechanisms to tolerate hypoxia (Centanin et al., 2008; Lee et al., 2019; Li et al., 2013). Here we show that induction of FOXO is one such mechanism and that it functions by regulating an immune-like response.

\section{RESULTS}

Hypoxia induces FOXO activity.

The main way that FOXO is regulated is through nuclear-cytoplasmic shuttling. In order to determine if hypoxia exposure could induce FOXO, we transferred third instar larvae growing on food to either moderate (5\% oxygen) or severe hypoxic environments (1\% oxygen) and then stained for FOXO localization using an anti-FOXO antibody (Figure 1A). We saw that exposure to hypoxia caused FOXO relocalization from the cytoplasm to the nucleus of fat body cells (Figure 1A). This effect was rapid; nuclear relocalization occured within 15 minutes of exposing larvae to hypoxia (Figure S1A). We next examined the effects of hypoxia on the expression of $4 e-b p$, a well-characterized FOXO target gene. We measured mRNA levels of $4 e-b p$ using qRT-PCR in whole third-instar larvae exposed to either $5 \%$ or $1 \%$ oxygen. We saw that $4 e-b p$ levels were strongly increased in control $\left(w^{1118}\right)$ larvae exposed to both hypoxic conditions (Figure 1B, C). As with the FOXO nuclear localization, this increase in $4 e-b p$ was rapid and was seen within 15-30 minutes following hypoxia exposure (Fig S1B). However, the hypoxia-induced increase in $4 e-b p$ mRNA levels was largely abolished in foxo $\triangle 94$, a deletion line that is a null mutant for the foxo gene (Slack et al., 2011)(Figure 1B, C). We also examined the effects of hypoxia in adults. We exposed adult females to $1 \% \mathrm{O}_{2}$ and found that, as in larvae, $4 e$-bp levels were increased in control $\left(w^{1118}\right)$ animals and that this effect was blunted in foxomutants (Figure 1D). Finally, we examined the tissue pattern of $4 e-b p$ induction by examining LacZ staining in thor-LacZ flies, which is a LacZ-enhancer trap in the 4e-bp gene locus (Bernal and Kimbrell, 2000). We found that larvae exposed to 2 hours of $5 \% \mathrm{O}_{2}$ showed increased LacZ staining in the majority of larval tissues including the fat body, the intestine, and the body wall muscle (Figure 1E), suggesting that the hypoxia induction of FOXO activity is not tissue-restricted. Together, these data indicate that exposure to hypoxia in both Drosophila larvae and adults results in rapid induction of FOXO transcriptional activity.

\section{FOXO is required for hypoxia tolerance.}

Is FOXO activation required for Drosophila survival in low oxygen? To find out, we measured hypoxia survival in foxo $\triangle 94$ animals. Under standard laboratory conditions (rich food, normoxia) foxo mutant animals are viable (Slack et al., 2011). We therefore examined how well these mutants tolerate low oxygen. We first examined hypoxia in larvae. Control $\left(w^{1118}\right)$ and foxo mutant embryos were allowed to 
develop in normoxia and then newly hatched larvae were transferred to hypoxia (5\% oxygen) for the duration of their larval period, before being returned to normoxia. We then counted the number of animals that developed to viable adults. We found that the foxo mutant animals reared in hypoxia had a significant decrease in viability compared to control animals (Figure 2A). We next examined hypoxia survival in adults. Control $\left(w^{1118}\right)$ and foxo mutant animals were exposed to either severe hypoxia ( $1 \%$ oxygen) for 24 hours or anoxia ( $0 \%$ oxygen) or 6 hours. After these low oxygen exposures, flies were returned to normoxia and the number of surviving animals counted. As observed in larvae, we found that the adult foxo mutant animals showed significantly deceased survival in both the hypoxic and anoxic conditions (Figure 2B, C). During severe hypoxia and anoxia, adult flies become immobile. However, when foxo adults were exposed to starvation instead of hypoxia for 24 hours, there was no effect on viability, indicating that the decrease in hypoxia survival in foxo mutants is not simply a consequence of reduced nutrient intake as a result of immobility (Fig S2). Together, our data indicate that FOXO activation is required for organismal survival in low oxygen in both developing larva and adults.

Cells, tissues and organisms adapt to low oxygen by altering their metabolism (Semenza, 2011). In particular, a key adaptation is the upregulation of glycolysis. We therefore checked whether FOXO might be important for controlling glucose metabolism in hypoxic animals. We first measured total glucose levels in adult animals exposed to hypoxia. Control animals exhibited a decrease in glucose levels after 16 hours of hypoxia (Figure 2D). foxo mutant flies had lower levels of total glucose in normoxia and these levels were even further depleted upon exposure to hypoxia (Figure 2D). We saw a similar pattern of effects when we measured levels of glycogen (the stored form of glucose) and trehalose (the circulating form of glucose in Drosophila). Thus, foxo mutants showed a significantly greater decrease in both glycogen and trehalose in hypoxia compared to control animals (Figure 2E, F).

Finally, we investigated expression of lactate dehydrogenase (Idh) - a key glycolytic enzyme - in $w^{1118}$ and foxo ${ }^{94}$ adult females. We saw that control animals increased their Idh mRNA when exposed to hypoxia as has been reported before (Lavista-Llanos et al., 2002; Li et al., 2013) and which is consistent with an upregulation of glycolysis. In contrast, foxo mutant animals had increased /dh levels in normoxia, and this expression increased significantly further in hypoxia (Figure 2G). Taken together, these data indicated that foxo mutants show deregulated control over normal glucose metabolism in hypoxia - they show overproduction of $I d h$ and they exhibit a larger depletion of both stored and circulating glucose in hypoxia compared to control animals.

\section{Hypoxia induces FOXO by inhibiting PI3K/Akt signalling.}


We next examined how hypoxia induces FOXO. The best-studied cellular response to hypoxia involves induction of the HIF1 $\alpha$ transcription factor (called sima in Drosophila). HIF $1 \alpha$ induces expression of metabolic and regulatory genes required for hypoxia adaptation, and in both Drosophila and $C$ elegans, $\mathrm{HIF} 1 \alpha$ is required for organismal tolerance to low oxygen (Centanin et al., 2005; Jiang et al., 2001). However, we found that FOXO was still relocalized to the nucleus in fat body cells from sima mutant larvae exposed to hypoxia (Fig 3A). This suggests that induction of FOXO is independent of the classic HIF1 $\alpha$ response.

One main way that FOXO can be regulated is via the conserved insulin/PI3K/Akt pathway (Webb and Brunet, 2014). This is best seen in response to nutrient availability in Drosophila. In rich nutrients, insulin signalling to Akt kinase is high and Akt can phosphorylate FOXO, leading its cytoplasmic retention. However, during starvation, insulin/Akt signalling is low, thus reducing phosphorylation of FOXO and allowing it to relocalize to the nucleus to induce transcription. We investigated whether decreased Akt activation was involved in FOXO induction during hypoxia exposure. Akt is activated by phosphorylation at two sites: threonine-342 and serine-505. We measured the relative amounts of Akt phosphorylated at each site after exposure to hypoxia using phospho-specific antibodies. We saw that when third instar larvae were exposed to hypoxia there was a reduction in phosphorylation of Akt at both sites (Figure 3B, C). To determine if suppression of Akt signalling was mediating the induction of FOXO, we used the flp-out technique to induce mosaic expression of the catalytic subunit of PI3K, $d p 110$, to maintain Akt activity in fat body cells. We found that during hypoxia, expression of $d p 110$ was sufficient to prevent FOXO nuclear relocalization (Figure 3D). Taken together, these data show that FOXO induction is mediated by hypoxia-induced suppression of Akt signalling.

\section{FOXO induces Relish-dependent hypoxia survival.}

In Drosophila, FOXO maintains tissue and organismal homeostasis in response to various stresses, including starvation, oxidative stress, irradiation, and infection. In each case, FOXO functions by regulating diverse and often distinct target genes. We surveyed potential FOXO targets that might be important for hypoxia tolerance and we identified a role for the NF-кB transcription factor relish.

In Drosophila there are three NF-кB transcription factors, Relish, Dorsal and Dif. They have been best characterized as effectors of immune signalling downstream of the IMD (Relish) and Toll (Dorsal and Dif) pathways, where they induce expression of antimicrobial peptides and promote innate immune responses (Buchon et al., 2014). We found that when exposed to hypoxia, adult Drosophila showed an increase in relish as reported previously (Bandarra et al., 2014; Liu et al., 2006), but not dorsal or dif, mRNA levels (Figure 4A-C). Furthermore, we found that this hypoxia-induced increase in relish was blocked in both foxo mutant adults (Figure 4D) and larvae (Figure S3). Finally, we found that hypoxia 
could induce strong expression of Relish-regulated antimicrobial peptides in both adults (Figure E, F) and larvae (Figure S3) and that this was also blocked in foxo mutants. These data suggest that in hypoxia, FOXO can induce an immune-like response via upregulation of Relish. To test whether this immune-like response was important for hypoxia survival, we examined hypoxia survival in two independent relish null mutants, $\mathrm{re}^{E 38}$ and $\mathrm{re}^{E 20}$ (Hedengren et al., 1999). We found that both re ${ }^{E 38}$ and rel $^{E 20}$ adult flies showed a significant decrease in viability after hypoxia exposure (Figure 4G, $H$ ). Together, these data point to FOXO activation as a meditator of hypoxia tolerance via induction of an immune-like response through the NFKB-like transcription factor relish.

\section{DISCUSSION}

In this paper, we report that FOXO is a hypoxia-inducible factor required for organismal survival in low oxygen. We saw that this induction of FOXO occurs via suppression of PI3K/Akt signalling. This response is most likely induced by hypoxia-mediated reduction of insulin release and signalling - the main activator of PI3K/Akt - as previously reported in Drosophila larvae (Texada et al., 2019; Wong et al., 2014). Interestingly we found that the induction of FOXO upon hypoxia occurs in sima mutants suggesting that the FOXO hypoxic response occurs independently of the classically described HIF-1 $\alpha$ response. Reduced insulin signalling and FOXO induction have been shown to confer hypoxia tolerance in C elegans (Mendenhall et al., 2006; Menuz et al., 2009; Scott et al., 2002). Moreover, the mammalian FOXO homolog FOXO3a can be induced in cell culture upon hypoxia exposure, where it regulates metabolic responses and cell death (Bakker et al., 2007; Jensen et al., 2011). Thus, the induction of FOXO is likely to be a conserved mechanism of hypoxia tolerance in animals.

A central finding of our work is that one way that FOXO provides protection in low oxygen is through induction of an immune-like response. In Drosophila, there are two main immune effector pathways that respond to pathogen infection and that work through induction of NF- $\mathrm{KB}$ transcription factors - the IMD pathway which targets the NF-кB homolog, Relish, and the Toll pathway which works via the Dorsal and Dif NF-KB transcription factors (Buchon et al., 2014). We found that hypoxia specifically induced Relish via FOXO, and that this response was required for hypoxia tolerance. These data, together with previous work showing hypoxia induction of Relish (Bandarra et al., 2014; Liu et al., 2006), suggest that induction of an immune-like response may be a protective mechanism in low oxygen in Drosophila. In the context of animal immunity, there is increasing appreciation of the role for infection tolerance as a defense strategy against pathogens (Ayres and Schneider, 2012; Lissner and Schneider, 2018; Medzhitov et al., 2012). This tolerance is often mediated via alterations in systemic metabolism and physiology to limit infection-induced tissue damage (Ganeshan et al., 2019; Wang et al., 2016; Weis et al., 2017). Our findings suggest that tolerance to hypoxia may share some of these immune functions. In Drosophila, this interplay between hypoxia and innate immune responses may reflect the natural 
ecology of flies. In the wild, Drosophila grow on rotting, fermenting food, an environment rich in microorganisms, including pathogenic bacteria. In these anaerobic conditions, low ambient oxygen may 'prime' animals to deal with subsequent pathogenic bacterial encounters. Hence, one speculative idea is that experimental exposure of Drosophila to hypoxia may induce Relish and provide protection against the detrimental effects of subsequent pathogenic infection. This concept of hypoxia preconditioning has been observed in $C$ elegans where it is important in protecting against cell death and damage induced by pore-forming toxins (Bellier et al., 2009; Dasgupta et al., 2007).

Functional interactions between FOXO and Relish have been described in response to other stressors in Drosophila. For example, nutrient starvation induces Relish in larvae via FOXO and this is important for controlling systemic insulin signalling (Karpac et al., 2011). In addition, as adults age, FOXO is induced in the intestine and it, in turn, upregulates Relish to control intestinal homeostasis and lifespan (Guo et al., 2014; Karpac et al., 2013). Interestingly, Relish and FOXO have an antagonistic relationship in adult fat and these interactions are important for metabolic adaption and survival upon starvation (Molaei et al., 2019). Hence the links between FOXO and relish are likely to be tissue specific, but they may have evolved to function as a general mediator of stress response. Functional links between NFКB and FOXO have also been reported in mammalian cells (Lin et al., 2004; Thompson et al., 2015),

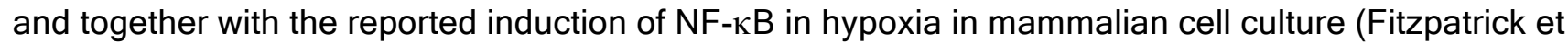
al., 2011; Rius et al., 2008), they suggest that the hypoxia-FOXO-NF-кB regulation that we see in Drosophila may operate in mammalian cells too.

One key way that cells, tissues and organisms adapt to low oxygen is by altering their glucose metabolism in order to maintain homeostasis (Nakazawa et al., 2016; Xie and Simon, 2017). Our data suggest that one reason that foxo mutants may show reduced hypoxia tolerance is that they have deregulated control over glucose metabolism. Thus, we saw that foxo mutant animals had low levels of glucose in normoxia and that both stored and circulating forms of glucose were significantly decreased under hypoxia compared to controls. These results suggest FOXO is needed for either gluconeogenesis during stress, as has been reported in $C$ elegans (Hibshman et al., 2017), or for proper control of glycolysis. Indeed, we saw that expression of $I d h$ is markedly increased in foxo mutants. Ldh is a rate-limiting enzyme involved in conversion of pyruvate to lactate, which is a key metabolic event that can drive increased glycolysis, and Idh levels have been shown to increase in larvae upon hypoxia exposure ( $\mathrm{Li}$ et al., 2013). Thus, one possibility is that foxo mutant animals may engage in abnormally high levels of glycolysis leading to depletion of glucose and reduced hypoxia tolerance. This is consistent with previous studies in Drosophila showing a major role for FOXO as a regulator of metabolic homeostasis in the context of other stress responses such as starvation and pathogenic infection (Dionne et al., 2006; Teleman et al., 2008). For example, FOXO often functions in 
a tissue specific manner to control systemic sugar and lipid metabolism (Borch Jensen et al., 2017; Karpac et al., 2013; Molaei et al., 2019; Wang et al., 2011; Zhao and Karpac, 2017). These effects have been shown to be important for FOXO to extend lifespan and to promote increased tolerance to stress.

It is possible that the effects of FOXO on metabolism in hypoxia could be mediated via Relish. For example, a recent report showed that Relish was required to control metabolic responses to nutrient deprivation in Drosophila (Molaei et al., 2019). Furthermore, constitutive activation of IMD signalling which signals via Relish - was shown to lead to decreased circulating sugars in adult Drosophila (Davoodi et al., 2019). In mammals, NF-кB is activated in response to cytokines and it functions as a central regulator of immune and inflammatory responses (Zhang et al., 2017). Several studies have

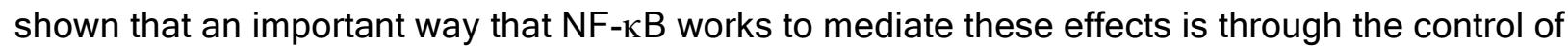
glycolysis and mitochondrial metabolic activity (Mauro et al., 2011; Tornatore et al., 2012). Indeed, links between immunity and metabolism are emerging as important components of infection tolerance in animals (Ayres and Schneider, 2012). Our data suggest the possibility that organisms may also co-opt some of these immune-metabolism interactions to tolerate low oxygen.

\section{METHODS AND MATERIALS}

\section{Drosophila stocks}

Flies were raised on standard medium containing $150 \mathrm{~g}$ agar, $1600 \mathrm{~g}$ cornmeal, $770 \mathrm{~g}$ Torula yeast, 675 g sucrose, $2340 \mathrm{~g} \mathrm{D}$-glucose, $240 \mathrm{ml}$ acid mixture (propionic acid/phosphoric acid) per $34 \mathrm{~L}$ water and maintained at $25^{\circ} \mathrm{C}$, unless otherwise indicated. The following fly stocks were used: $w^{1118}$, sima ${ }^{07607} / T M 3$, Ser, GFP (Centanin et al., 2008), foxo ${ }^{94} / T M 3$, Ser (Slack et al., 2011), Thor-LacZ (Bernal and Kimbrell, 2000), hsflp; UAS-dp110, act>CD2>Gal4,UAS-GFP (Britton et al., 2002), Relish $^{E 20}$ (Hedengren et al., 1999), Relish ${ }^{E 38}$ (Hedengren et al., 1999).

\section{Hypoxia exposure}

For all hypoxia experiments vials containing Drosophila were placed into an airtight glass chamber into which a premix of $5 \%$ oxygen/95\% nitrogen, $1 \%$ oxygen/99\%nitrogen or $100 \%$ nitrogen continually flowed. Flow rate was controlled using an Aalborg model P gas flow meter. Alternatively, for some experiments Drosophila vials were placed into a Coy Laboratory Products in vitro $\mathrm{O}_{2}$ chamber that was maintained at fixed oxygen levels of $1 \%$ or $5 \%$ by injection of nitrogen gas.

\section{Immunofluorescence staining}


Larvae were inverted using fine forceps in 1x PBS. Inverted larvae were fixed in $8 \%$ paraformaldehyde for 30 minutes, washed in 1x PBS/0.1\% TritonX-100 (PBST), and blocked for 2 hours at room temperature in 1x PBS/0.1\%Tween20/1\% bovine serum albumin. Larvae were then incubated overnight with primary antibody diluted in PAT at $4^{\circ} \mathrm{C}$, washed 3 times with $1 \mathrm{x}$ PBS with $3 \%$ TritonX-100 (PBT) and $2 \%$ fetal bovine serum (FBS), and incubated with secondary antibody diluted 1:4000 in PBT with FBS for 2 hours at room temperature. Larvae were washed with PBT and stained with 1:10000 Hoechst dye for 5 minutes, then washed 3 times more with PBT. Larval tissues were isolated using fine forceps and then mounted on glass slides with cover slips using Vectashield mounting media (Vector Laboratories Inc., CA). The rabbit anti-FOXO antibody was used at 1:500 dilution (a gift from Marc Tatar). Alexa Fluor 568 (Invitrogen) was used as the secondary antibody. Hoechst 33342 (Invitrogen) was used to stain nuclei.

\section{Quantitative PCR}

Total RNA was extracted using TRIzol according to manufacturer's instructions (Invitrogen; 15596-018). RNA samples were then subjected to DNase treatment according to manufacturer's instructions (Ambion; $2238 \mathrm{G}$ ) and reverse transcribed using Superscript II (Invitrogen; 100004925). The generated cDNA was used as a template to perform qRT-PCRs (ABI 7500 real time PCR system using SyBr Green PCR mix) using specific primer pairs. PCR data were normalized to beta-tubulin levels. Each experiment was independently repeated a minimum of three times. The following primers were used:

beta-tubulin: Forward 5' ATCATCACACACGGACAGG; Reverse 5' GAGCTGGATGATGGGGAGTA 4e-bp: Forward 5' GCTAAGATGTCCGCTTCACC; Reverse: 5' CCTCCAGGAGTGGTGGAGTA relish: Forward 5' TCCTTAATGGAGTGCCAACC; Reverse 5' TGCCATGTGGAGTGCATTAT dorsal: Forward 5' TGTTCAAATCGCGGGCGTCGA; Reverse 5' TCGGACACCTTCGAGCTCCAGAA dif: Forward 5' CGGACGTGAAGCGCCGACTTG; Reverse 5' CAGCCGCCTGTTTAGAGCGG attacin A: Forward 5' AGGAGGCCCATGCCAATTTA; Reverse 5' CATTCCGCTGGAACTCGAAA cecropin A: Forward 5' TCTTCGTTTTCGTCGCTCTCA; Reverse 5' ATTCCCAGTCCCTGGATTGTG

\section{Lac Z staining}

Larvae were inverted using fine forceps in 1x PBS. Inverted larvae were fixed in $8 \%$ paraformaldehyde for 30 minutes, washed in 1x PBS-0.1\% TritonX-100 (PBST), and then incubated in 500 $\mu$ l of an X-Gal solution containing10 mM sodium phosphate buffer, $\mathrm{pH} 7.2,150 \mathrm{mM} \mathrm{NaCl}, 1 \mathrm{mM} \mathrm{MgCl} 2,10 \mathrm{mM}$

$\mathrm{K}_{4}\left[\mathrm{Fe}^{\mathrm{II}}(\mathrm{CN})_{6}\right], 10 \mathrm{mM} \mathrm{K}_{3}\left[\mathrm{Fe}^{\mathrm{III}}(\mathrm{CN})_{6}\right], 0.1 \%$ Triton $\mathrm{X}-100$ with $12.5 \mu$ l of an $8 \% \mathrm{X}$-Gal solution (in DMSO) added immediately prior to incubation. Samples were then incubated at 37C until the X-Gal staining was visible. 


\section{Measurement of hypoxia survival}

Larvae: newly hatched larvae were placed in food vials (50 larvae per vial) and then maintained in either normoxia or hypoxia (5\% oxygen). Larvae exposed to hypoxia were maintained in this environment until about $80 \%$ of larvae had pupated. Then, vials were removed from hypoxia and the numbers of eclosing adults were counted.

Adults:. 4-5 days post-eclosion, mated female adults were placed in placed into hypoxia (1\% oxygen) for 24 hours in cohorts of 20 flies per vial. Then, vials were removed from hypoxia and the flies were allowed to recover for 48 hours before the number of dead flies were counted.

Starvation: At 4-5 days post-eclosion, mated female adults were subjected to starvation by transferring them from food vials to vials containing $0.4 \%$ agar/PBS for 24 hours. The number of dead flies was then counted.

\section{Glucose, glycogen, trehalose and TAG assays}

Adult female Drosophila were either exposed to hypoxia (1\% oxygen) for 16 hours or maintained in normoxia and then frozen on dry ice. Colorimetric assays for each of the metabolites were then conducted using the methods described in detail in (Tennessen et al., 2014).

\section{Preparation of protein extracts and western blotting}

Drosophila larvae were lysed with a buffer containing $20 \mathrm{mM}$ Tris- $\mathrm{HCl}(\mathrm{pH} 8.0), 137 \mathrm{mM} \mathrm{NaCl}, 1 \mathrm{mM}$ EDTA, $25 \%$ glycerol, 1\% NP-40 and with following inhibitors 50 mM NaF, 1 mM PMSF, 1 mM DTT, 5 $\mathrm{mM}$ sodium ortho vanadate $\left(\mathrm{Na}_{3} \mathrm{VO}_{4}\right)$ and Protease Inhibitor cocktail (Roche Cat. No. 04693124001) and Phosphatase inhibitor (Roche Cat. No. 04906845001), according to the manufacturer instructions. Protein concentrations were measured using the Bio-Rad Dc Protein Assay kit II (5000112). Protein lysates $(15 \mu \mathrm{g}$ to $30 \mu \mathrm{g}$ ) were resolved by SDS-PAGE and electro transferred to a nitrocellulose membrane, subjected to Western blot analysis with specific antibodies, and visualized by chemiluminescence (enhanced ECL solution (Perkin Elmer)). Primary antibodies used in this study were: anti-Akt (Cell Signaling \#9272, 1:500 dilution), anti-pAkt-T342 (gift from Michelle Bland), antipAkt-S505 (Cell Signaling \#4054, 1:1000 dilution). Secondary antibodies were purchased from SantaCruz Biotechnology (sc-2030, 2005, 2020). For experiments looking at Akt phosphorylation, total Akt levels were used as a loading control because the level of this protein was unaffected by hypoxia.

\section{Statistical analyses}


Data were analyzed by Students t-test or two-way ANOVA. All statistical analysis and data plots were performed using Prism software. In all figures, statistically significant differences are presented as: * and indicate $\mathrm{p}<0.05$.

\section{ACKNOWLEDGEMENTS}

We thank Edan Foley, Linda Partridge, Bruce Edgar, Mark Tatar for the gift of reagents and fly stocks. Stocks obtained from the Bloomington Drosophila Stock Center (NIH P40OD018537) were used in this study. This work was supported by a NSERC Discovery grant to S.S.G. E.C.B was supported by an Alberta Innovates Health Solutions Graduate Studentship. A.N.B-P was supported by an NSERC summer studentship. D.M.P was supported by an NSERC CGS-M graduate scholarship. 


\section{REFERENCES}

Alic, N., Andrews, T.D., Giannakou, M.E., Papatheodorou, I., Slack, C., Hoddinott, M.P., Cocheme, H.M., Schuster, E.F., Thornton, J.M., and Partridge, L. (2011). Genome-wide dFOXO targets and topology of the transcriptomic response to stress and insulin signalling. Mol Syst Biol 7, 502.

Alic, N., Tullet, J.M., Niccoli, T., Broughton, S., Hoddinott, M.P., Slack, C., Gems, D., and Partridge, L. (2014). Cell-nonautonomous effects of dFOXO/DAF-16 in aging. Cell reports 6, 608-616.

Ayres, J.S., and Schneider, D.S. (2012). Tolerance of infections. Annu Rev Immunol 30, 271-294.

Bakker, W.J., Harris, I.S., and Mak, T.W. (2007). FOXO3a is activated in response to hypoxic stress and inhibits HIF1-induced apoptosis via regulation of CITED2. Mol Cell 28, 941-953.

Bandarra, D., Biddlestone, J., Mudie, S., Muller, H.A., and Rocha, S. (2014). Hypoxia activates IKK-NFkappaB and the immune response in Drosophila melanogaster. Biosci Rep 34.

Bellier, A., Chen, C.S., Kao, C.Y., Cinar, H.N., and Aroian, R.V. (2009). Hypoxia and the hypoxic response pathway protect against pore-forming toxins in C. elegans. PLoS Pathog 5, e1000689.

Bernal, A., and Kimbrell, D.A. (2000). Drosophila Thor participates in host immune defense and connects a translational regulator with innate immunity. Proc Natl Acad Sci U S A 97, 6019-6024.

Birnbaum, A., Wu, X., Tatar, M., Liu, N., and Bai, H. (2019). Age-Dependent Changes in Transcription Factor FOXO Targeting in Female Drosophila. Front Genet 10, 312.

Borch Jensen, M., Qi, Y., Riley, R., Rabkina, L., and Jasper, H. (2017). PGAM5 promotes lasting FoxO activation after developmental mitochondrial stress and extends lifespan in Drosophila. eLife 6.

Boutin, A.T., Weidemann, A., Fu, Z., Mesropian, L., Gradin, K., Jamora, C., Wiesener, M., Eckardt, K.U., Koch, C.J., Ellies, L.G., et al. (2008). Epidermal sensing of oxygen is essential for systemic hypoxic response. Cell 133, 223-234.

Britton, J.S., Lockwood, W.K., Li, L., Cohen, S.M., and Edgar, B.A. (2002). Drosophila's insulin/PI3kinase pathway coordinates cellular metabolism with nutritional conditions. Developmental cell 2, 239249.

Buchon, N., Silverman, N., and Cherry, S. (2014). Immunity in Drosophila melanogaster--from microbial recognition to whole-organism physiology. Nat Rev Immunol 14, 796-810.

Callier, V., Hand, S.C., Campbell, J.B., Biddulph, T., and Harrison, J.F. (2015). Developmental changes in hypoxic exposure and responses to anoxia in Drosophila melanogaster. J Exp Biol 218, 2927-2934. 
Centanin, L., Dekanty, A., Romero, N., Irisarri, M., Gorr, T.A., and Wappner, P. (2008). Cell autonomy of HIF effects in Drosophila: tracheal cells sense hypoxia and induce terminal branch sprouting.

Developmental cell 14, 547-558.

Centanin, L., Ratcliffe, P.J., and Wappner, P. (2005). Reversion of lethality and growth defects in Fatiga oxygen-sensor mutant flies by loss of hypoxia-inducible factor-alpha/Sima. EMBO Rep 6, 1070-1075.

Cramer, T., Yamanishi, Y., Clausen, B.E., Forster, I., Pawlinski, R., Mackman, N., Haase, V.H., Jaenisch, R., Corr, M., Nizet, V., et al. (2003). HIF-1alpha is essential for myeloid cell-mediated inflammation. Cell 112, 645-657.

Dasgupta, N., Patel, A.M., Scott, B.A., and Crowder, C.M. (2007). Hypoxic preconditioning requires the apoptosis protein CED-4 in C. elegans. Current biology : CB 17, 1954-1959.

Davoodi, S., Galenza, A., Panteluk, A., Deshpande, R., Ferguson, M., Grewal, S., and Foley, E. (2019). The Immune Deficiency Pathway Regulates Metabolic Homeostasis in Drosophila. J Immunol 202, 2747-2759.

Demontis, F., and Perrimon, N. (2010). FOXO/4E-BP signaling in Drosophila muscles regulates organism-wide proteostasis during aging. Cell 143, 813-825.

Dionne, M.S., Pham, L.N., Shirasu-Hiza, M., and Schneider, D.S. (2006). Akt and FOXO dysregulation contribute to infection-induced wasting in Drosophila. Current biology : CB 16, 1977-1985.

Fitzpatrick, S.F., Tambuwala, M.M., Bruning, U., Schaible, B., Scholz, C.C., Byrne, A., O'Connor, A., Gallagher, W.M., Lenihan, C.R., Garvey, J.F., et al. (2011). An intact canonical NF-kappaB pathway is required for inflammatory gene expression in response to hypoxia. J Immunol 186, 1091-1096.

Ganeshan, K., Nikkanen, J., Man, K., Leong, Y.A., Sogawa, Y., Maschek, J.A., Van Ry, T., Chagwedera, D.N., Cox, J.E., and Chawla, A. (2019). Energetic Trade-Offs and Hypometabolic States Promote Disease Tolerance. Cell 177, 399-413 e312.

Gershman, B., Puig, O., Hang, L., Peitzsch, R.M., Tatar, M., and Garofalo, R.S. (2007). High-resolution dynamics of the transcriptional response to nutrition in Drosophila: a key role for dFOXO. Physiol Genomics 29, 24-34.

Giannakou, M.E., Goss, M., Junger, M.A., Hafen, E., Leevers, S.J., and Partridge, L. (2004). Long-lived Drosophila with overexpressed dFOXO in adult fat body. Science (New York, N.Y.) 305, 361. 
Guo, L., Karpac, J., Tran, S.L., and Jasper, H. (2014). PGRP-SC2 promotes gut immune homeostasis to limit commensal dysbiosis and extend lifespan. Cell 156, 109-122.

Harrison, J.F., Greenlee, K.J., and Verberk, W. (2018). Functional Hypoxia in Insects: Definition, Assessment, and Consequences for Physiology, Ecology, and Evolution. Annu Rev Entomol 63, 303325.

Hedengren, M., Asling, B., Dushay, M.S., Ando, I., Ekengren, S., Wihlborg, M., and Hultmark, D. (1999). Relish, a central factor in the control of humoral but not cellular immunity in Drosophila. Mol Cell 4, 827837.

Hibshman, J.D., Doan, A.E., Moore, B.T., Kaplan, R.E., Hung, A., Webster, A.K., Bhatt, D.P., Chitrakar, R., Hirschey, M.D., and Baugh, L.R. (2017). daf-16/FoxO promotes gluconeogenesis and trehalose synthesis during starvation to support survival. eLife 6 .

Huang, Y., Hickey, R.P., Yeh, J.L., Liu, D., Dadak, A., Young, L.H., Johnson, R.S., and Giordano, F.J. (2004). Cardiac myocyte-specific HIF-1alpha deletion alters vascularization, energy availability, calcium flux, and contractility in the normoxic heart. FASEB J 18, 1138-1140.

Hwangbo, D.S., Gersham, B., Tu, M.P., Palmer, M., and Tatar, M. (2004). Drosophila dFOXO controls lifespan and regulates insulin signalling in brain and fat body. Nature 429, 562-566.

Jensen, K.S., Binderup, T., Jensen, K.T., Therkelsen, I., Borup, R., Nilsson, E., Multhaupt, H., Bouchard, C., Quistorff, B., Kjaer, A., et al. (2011). FoxO3A promotes metabolic adaptation to hypoxia by antagonizing Myc function. EMBO J 30, 4554-4570.

Jiang, H., Guo, R., and Powell-Coffman, J.A. (2001). The Caenorhabditis elegans hif-1 gene encodes a bHLH-PAS protein that is required for adaptation to hypoxia. Proc Natl Acad Sci U S A 98, 7916-7921.

Junger, M.A., Rintelen, F., Stocker, H., Wasserman, J.D., Vegh, M., Radimerski, T., Greenberg, M.E., and Hafen, E. (2003). The Drosophila forkhead transcription factor FOXO mediates the reduction in cell number associated with reduced insulin signaling. J Biol 2, 20.

Karpac, J., Biteau, B., and Jasper, H. (2013). Misregulation of an adaptive metabolic response contributes to the age-related disruption of lipid homeostasis in Drosophila. Cell reports 4, 1250-1261.

Karpac, J., Hull-Thompson, J., Falleur, M., and Jasper, H. (2009). JNK signaling in insulin-producing cells is required for adaptive responses to stress in Drosophila. Aging Cell 8, 288-295. 
Karpac, J., Younger, A., and Jasper, H. (2011). Dynamic coordination of innate immune signaling and insulin signaling regulates systemic responses to localized DNA damage. Developmental cell 20, 841 854.

Kramer, J.M., Slade, J.D., and Staveley, B.E. (2008). foxo is required for resistance to amino acid starvation in Drosophila. Genome 51, 668-672.

Lavista-Llanos, S., Centanin, L., Irisarri, M., Russo, D.M., Gleadle, J.M., Bocca, S.N., Muzzopappa, M., Ratcliffe, P.J., and Wappner, P. (2002). Control of the hypoxic response in Drosophila melanogaster by the basic helix-loop-helix PAS protein similar. Mol Cell Biol 22, 6842-6853.

Lee, B., Barretto, E.C., and Grewal, S.S. (2019). TORC1 modulation in adipose tissue is required for organismal adaptation to hypoxia in Drosophila. Nat Commun 10, 1878.

Li, Y., Padmanabha, D., Gentile, L.B., Dumur, C.I., Beckstead, R.B., and Baker, K.D. (2013). HIF- and non-HIF-regulated hypoxic responses require the estrogen-related receptor in Drosophila melanogaster. PLoS genetics 9, e1003230.

Lin, L., Hron, J.D., and Peng, S.L. (2004). Regulation of NF-kappaB, Th activation, and autoinflammation by the forkhead transcription factor Foxo3a. Immunity 21, 203-213.

Lissner, M.M., and Schneider, D.S. (2018). The physiological basis of disease tolerance in insects. Curr Opin Insect Sci 29, 133-136.

Liu, G., Roy, J., and Johnson, E.A. (2006). Identification and function of hypoxia-response genes in Drosophila melanogaster. Physiol Genomics 25, 134-141.

Markow, T.A. (2015). The secret lives of Drosophila flies. eLife 4.

Mason, S.D., Howlett, R.A., Kim, M.J., Olfert, I.M., Hogan, M.C., McNulty, W., Hickey, R.P., Wagner, P.D., Kahn, C.R., Giordano, F.J., et al. (2004). Loss of skeletal muscle HIF-1alpha results in altered exercise endurance. PLoS Biol 2, e288.

Mauro, C., Leow, S.C., Anso, E., Rocha, S., Thotakura, A.K., Tornatore, L., Moretti, M., De Smaele, E., Beg, A.A., Tergaonkar, V., et al. (2011). NF-kappaB controls energy homeostasis and metabolic adaptation by upregulating mitochondrial respiration. Nat Cell Biol 13, 1272-1279.

McKeown, S.R. (2014). Defining normoxia, physoxia and hypoxia in tumours-implications for treatment response. The British journal of radiology 87, 20130676. 
Medzhitov, R., Schneider, D.S., and Soares, M.P. (2012). Disease tolerance as a defense strategy. Science (New York, N.Y.) 335, 936-941.

Mendenhall, A.R., LaRue, B., and Padilla, P.A. (2006). Glyceraldehyde-3-phosphate dehydrogenase mediates anoxia response and survival in Caenorhabditis elegans. Genetics 174, 1173-1187.

Menuz, V., Howell, K.S., Gentina, S., Epstein, S., Riezman, I., Fornallaz-Mulhauser, M., Hengartner, M.O., Gomez, M., Riezman, H., and Martinou, J.C. (2009). Protection of C. elegans from anoxia by HYL-2 ceramide synthase. Science (New York, N.Y.) 324, 381-384.

Molaei, M., Vandehoef, C., and Karpac, J. (2019). NF-kappaB Shapes Metabolic Adaptation by Attenuating Foxo-Mediated Lipolysis in Drosophila. Developmental cell 49, 802-810 e806.

Nakazawa, M.S., Keith, B., and Simon, M.C. (2016). Oxygen availability and metabolic adaptations. Nat Rev Cancer 16, 663-673.

Rius, J., Guma, M., Schachtrup, C., Akassoglou, K., Zinkernagel, A.S., Nizet, V., Johnson, R.S., Haddad, G.G., and Karin, M. (2008). NF-kappaB links innate immunity to the hypoxic response through transcriptional regulation of HIF-1alpha. Nature 453, 807-811.

Schipani, E., Ryan, H.E., Didrickson, S., Kobayashi, T., Knight, M., and Johnson, R.S. (2001). Hypoxia in cartilage: HIF-1alpha is essential for chondrocyte growth arrest and survival. Genes Dev 15, 28652876.

Scott, B.A., Avidan, M.S., and Crowder, C.M. (2002). Regulation of hypoxic death in C. elegans by the insulin/IGF receptor homolog DAF-2. Science (New York, N.Y.) 296, 2388-2391.

Semenza, G.L. (2011). Oxygen sensing, homeostasis, and disease. N Engl J Med 365, 537-547.

Semenza, G.L. (2014). Oxygen sensing, hypoxia-inducible factors, and disease pathophysiology. Annu Rev Pathol 9, 47-71.

Slack, C., Giannakou, M.E., Foley, A., Goss, M., and Partridge, L. (2011). dFOXO-independent effects of reduced insulin-like signaling in Drosophila. Aging Cell 10, 735-748.

Teleman, A.A., Hietakangas, V., Sayadian, A.C., and Cohen, S.M. (2008). Nutritional control of protein biosynthetic capacity by insulin via Myc in Drosophila. Cell metabolism 7, 21-32.

Tennessen, J.M., Barry, W.E., Cox, J., and Thummel, C.S. (2014). Methods for studying metabolism in Drosophila. Methods 68, 105-115. 
Texada, M.J., Jorgensen, A.F., Christensen, C.F., Koyama, T., Malita, A., Smith, D.K., Marple, D.F.M., Danielsen, E.T., Petersen, S.K., Hansen, J.L., et al. (2019). A fat-tissue sensor couples growth to oxygen availability by remotely controlling insulin secretion. Nat Commun 10, 1955.

Thompson, M.G., Larson, M., Vidrine, A., Barrios, K., Navarro, F., Meyers, K., Simms, P., Prajapati, K., Chitsike, L., Hellman, L.M., et al. (2015). FOXO3-NF-kappaB RelA Protein Complexes Reduce Proinflammatory Cell Signaling and Function. J Immunol 195, 5637-5647.

Tomita, S., Ueno, M., Sakamoto, M., Kitahama, Y., Ueki, M., Maekawa, N., Sakamoto, H., Gassmann, M., Kageyama, R., Ueda, N., et al. (2003). Defective brain development in mice lacking the Hif-1alpha gene in neural cells. Mol Cell Biol 23, 6739-6749.

Tornatore, L., Thotakura, A.K., Bennett, J., Moretti, M., and Franzoso, G. (2012). The nuclear factor kappa B signaling pathway: integrating metabolism with inflammation. Trends in cell biology 22, 557566.

Wang, A., Huen, S.C., Luan, H.H., Yu, S., Zhang, C., Gallezot, J.D., Booth, C.J., and Medzhitov, R. (2016). Opposing Effects of Fasting Metabolism on Tissue Tolerance in Bacterial and Viral Inflammation. Cell 166, 1512-1525 e1512.

Wang, B., Moya, N., Niessen, S., Hoover, H., Mihaylova, M.M., Shaw, R.J., Yates, J.R., 3rd, Fischer, W.H., Thomas, J.B., and Montminy, M. (2011). A hormone-dependent module regulating energy balance. Cell 145, 596-606.

Webb, A.E., and Brunet, A. (2014). FOXO transcription factors: key regulators of cellular quality control. Trends Biochem Sci 39, 159-169.

Weis, S., Carlos, A.R., Moita, M.R., Singh, S., Blankenhaus, B., Cardoso, S., Larsen, R., Rebelo, S., Schauble, S., Del Barrio, L., et al. (2017). Metabolic Adaptation Establishes Disease Tolerance to Sepsis. Cell 169, 1263-1275 e1214.

Wong, D.M., Shen, Z., Owyang, K.E., and Martinez-Agosto, J.A. (2014). Insulin- and warts-dependent regulation of tracheal plasticity modulates systemic larval growth during hypoxia in Drosophila melanogaster. PLoS One 9, e115297.

Xie, H., and Simon, M.C. (2017). Oxygen availability and metabolic reprogramming in cancer. The Journal of biological chemistry 292, 16825-16832.

Zhang, Q., Lenardo, M.J., and Baltimore, D. (2017). 30 Years of NF-kappaB: A Blossoming of Relevance to Human Pathobiology. Cell 168, 37-57. 
Zhao, X., and Karpac, J. (2017). Muscle Directs Diurnal Energy Homeostasis through a MyokineDependent Hormone Module in Drosophila. Current biology : CB 27, 1941-1955 e1946.

\section{FIGURE LEGENDS}

Figure 1. Hypoxia induces FOXO activity. (A) FOXO staining of 96-hour AEL $w^{1118}$ larval fat bodies following exposure to hypoxia for two hours. Nuclei are stained with Hoechst (bottom panels). Scale bar is $25 \mu \mathrm{m}$. (B) $4 e-b p$ mRNA levels measured by qRT-PCR in control $\left(w^{1118}\right)$ and foxo mutant (foxo ${ }^{94}$ ) following B) 6 hours of $5 \% \mathrm{O}_{2}$ hypoxia in larvae, C) 6 hours of $1 \% \mathrm{O}_{2}$ hypoxia in larvae, or D) 16 hours of $1 \% \mathrm{O}_{2}$ hypoxia in adults. $\mathrm{N}>6$ cohorts of animals per condition. Data represent mean + SEM. * p,0.05, two-way ANOVA followed by post-hoc t-test. (E) LacZ staining in tissues of thor-LacZ larvae following two-hour exposure to $5 \% \mathrm{O}_{2}$. Scale bar is $100 \mu \mathrm{m}$.

Figure 2. FOXO is required for hypoxia tolerance. (A) Control ( $w^{1118}$ ) and foxo mutant (foxo $\left.{ }^{94}\right)$ animals were exposed to hypoxia $\left(5 \% \mathrm{O}_{2}\right)$ throughout their larval period, before being returned to normoxia as pupae. The percentage of flies that eclosed as viable adults were then counted. $(B, C)$ Adult control $\left(w^{1118}\right)$ or foxo mutant $\left(\right.$ foxo $\left.{ }^{94}\right)$ flies were exposed to either, $\left.\mathrm{B}\right) 24$ hours of $1 \% \mathrm{O}_{2}$ or C) 6 hours of $0 \% \mathrm{O}_{2}$, before being returned to normoxia. The percentage of viable flies was then counted. Data represent mean + SEM. ${ }^{*} p<0.05$, students t-test. $N>4$ cohorts of animals per condition. (D-F) Relative levels of free glucose $(\mathrm{D})$, glycogen $(\mathrm{E})$, or trehalose $(\mathrm{F})$, in adult control $\left(w^{1118}\right)$ and foxo mutant $\left(\right.$ foxo $^{94}$ ) flies exposed to normoxia or $1 \% \mathrm{O}_{2}$ hypoxia for 16 hours. $\mathrm{n}=15$. Data represents mean + SEM. * ${ }^{*}<0.05$, students t-test. (G) Ldh mRNA levels measured by qRT-PCR in control $\left(w^{1118}\right)$ and foxo mutant $\left(\right.$ foxo ${ }^{94}$ ) following 16 hours of $1 \% \mathrm{O}_{2}$ hypoxia in adults. Data represent mean + SEM. * p,0.05, two-way ANOVA followed by post-hoc t-test. N>10 per condition.

Figure 3. Hypoxia induces FOXO by inhibiting PI3K/Akt. (A) FOXO staining in fat bodies of control $\left(w^{1118}\right)$ and sima mutant (sima ${ }^{07607}$ ) larvae exposed to either normoxia or $5 \% \mathrm{O}_{2}$ hypoxia for 2 hours. Scale bar is $25 \mu \mathrm{m}$. (B,C) Western blot analysis of phosphorylated T342 and S505 Akt, and total Akt in control $\left(w^{1118}\right)$ larvae following 2 hours of normoxia $(\mathrm{N})$ or $5 \% \mathrm{O}_{2}$ hypoxia. Quantification of blots (relative phospho-Akt intensity/total Akt intensity) is shown in (D). N=4 per condition. * $p<0.05$, students t-test. (D) FOXO staining in UAS- $d p 110$ overexpressing fat body clones (GFP positive). Nuclei are stained with Hoechst dye (blue). Scale bar is $50 \mu \mathrm{m}$.

Figure 4. FOXO induces Relish-dependent hypoxia survival. (A-C) Expression levels of relish $(\mathrm{A}), \operatorname{dif}(\mathrm{B})$, and dorsal $(\mathrm{C}) \mathrm{mRNA}$ in $w^{1118}$ adult females exposed to either normoxia or 16 hours of $1 \%$ 
$\mathrm{O}_{2}$. Data represent mean $+\mathrm{SEM}, \mathrm{N}=10,{ }^{*} \mathrm{p}<0.05$, students t-test. (D-F) Expression levels of relish (D), attacin $A(E)$, and cecropin $A(F)$ mRNA in $w^{1118}$ and foxo ${ }^{94}$ adult females exposed to either normoxia or 16 hours of $1 \% \mathrm{O}_{2}$. Data represent mean + SEM, N=10, * $\mathrm{p}<0.05,2$-way ANOVA followed by students ttest. $(\mathrm{G}, \mathrm{H})$ Survival of adult female $w^{1118}$ or $(\mathrm{G})$ relish $^{E 38}$ or $(\mathrm{H})$ relish ${ }^{E 20}$ flies after exposure to 24 hours of $1 \% \mathrm{O}_{2}$. Data represents mean $+\mathrm{SEM}, \mathrm{N}={ }^{*} \mathrm{p}<0.05$, students t-test.

Figure S1. FOXO is induced rapidly in hypoxia. (A) FOXO staining of 96-hour AEL $w^{1118}$ larval fat bodies following exposure to hypoxia for 15 minutes. Nuclei are stained with Hoechst (bottom panels). Scale bar is $25 \mu \mathrm{m}$. (B) $4 e-b p$ mRNA levels measured by qRT-PCR in control ( $w^{1118}$ ) larvae exposed to either normoxia or hypoxia (1\% oxygen) for 15 or 30 minutes. Data represent mean $+\mathrm{SEM}$, $\mathrm{N}=10,{ }^{*} \mathrm{p}<0.05$, students t-test.

Figure S2. foxo mutant survival is not affected by short term nutrient deprivation. (A) Survival of adult female $w^{1118}$ and foxo ${ }^{94}$ flies 2 days after starvation for 24 hours. Data represented as mean + SEM for $n=4$ groups of 20 flies.

Figure S3. relish is induced by FOXO in hypoxic larvae. Expression levels of $(A)$ relish or (B) attacin $\mathrm{A}$ mRNA in $w^{1118}$ and foxo ${ }^{94}$ larvae exposed to $5 \% \mathrm{O}_{2}$ for 6 hours. Data represent mean + SEM, $\mathrm{N}=10,{ }^{*} \mathrm{p}<0.05,2$-way ANOVA followed by students t-test. 
A
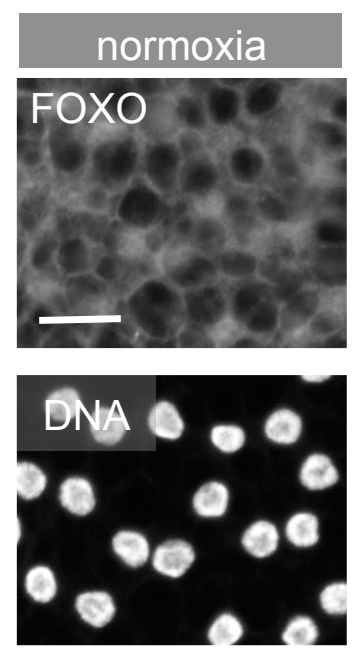
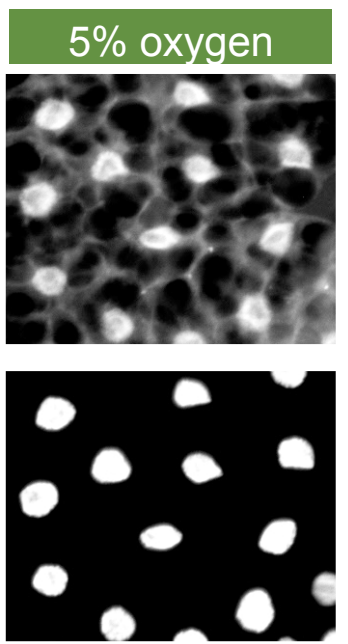
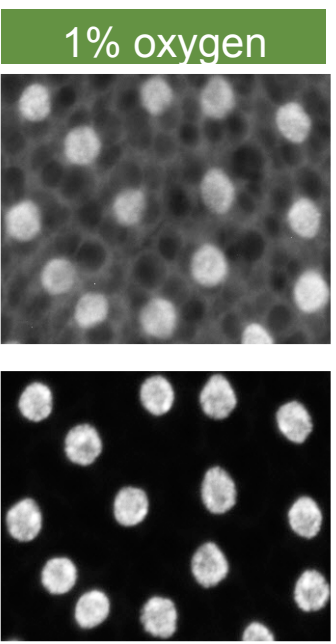

B

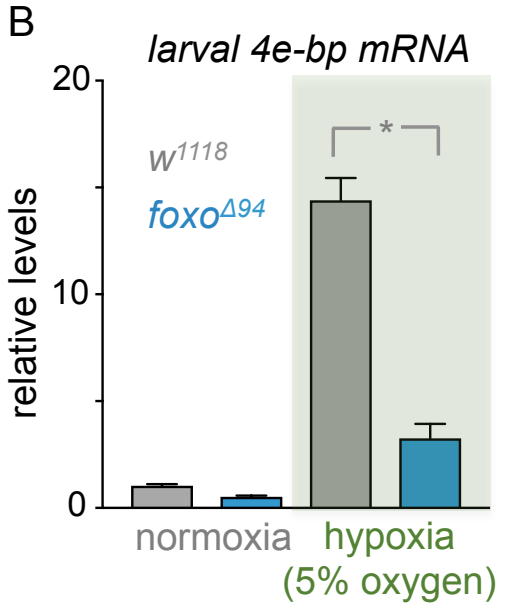

C
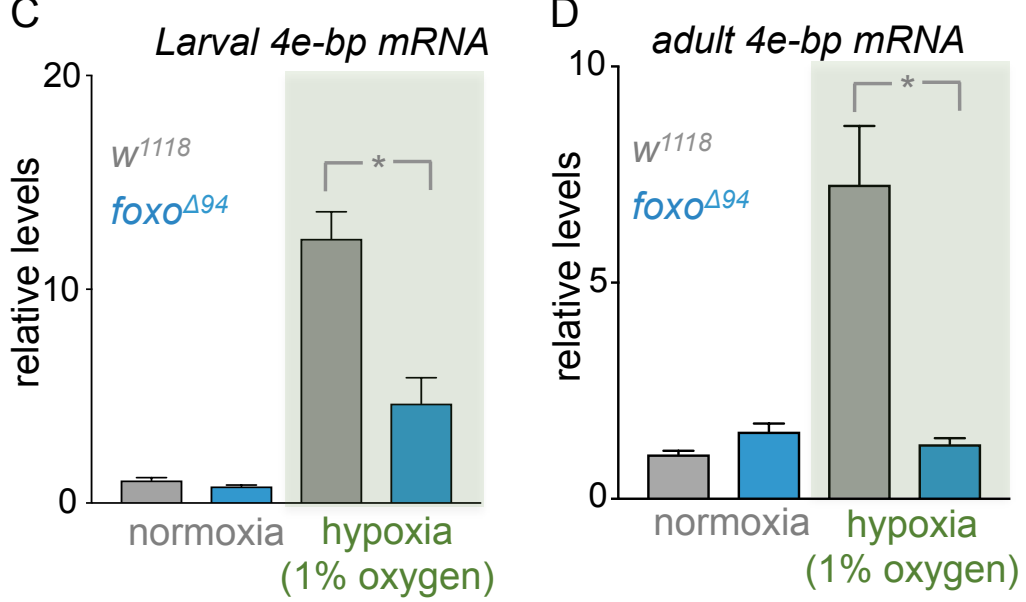

E
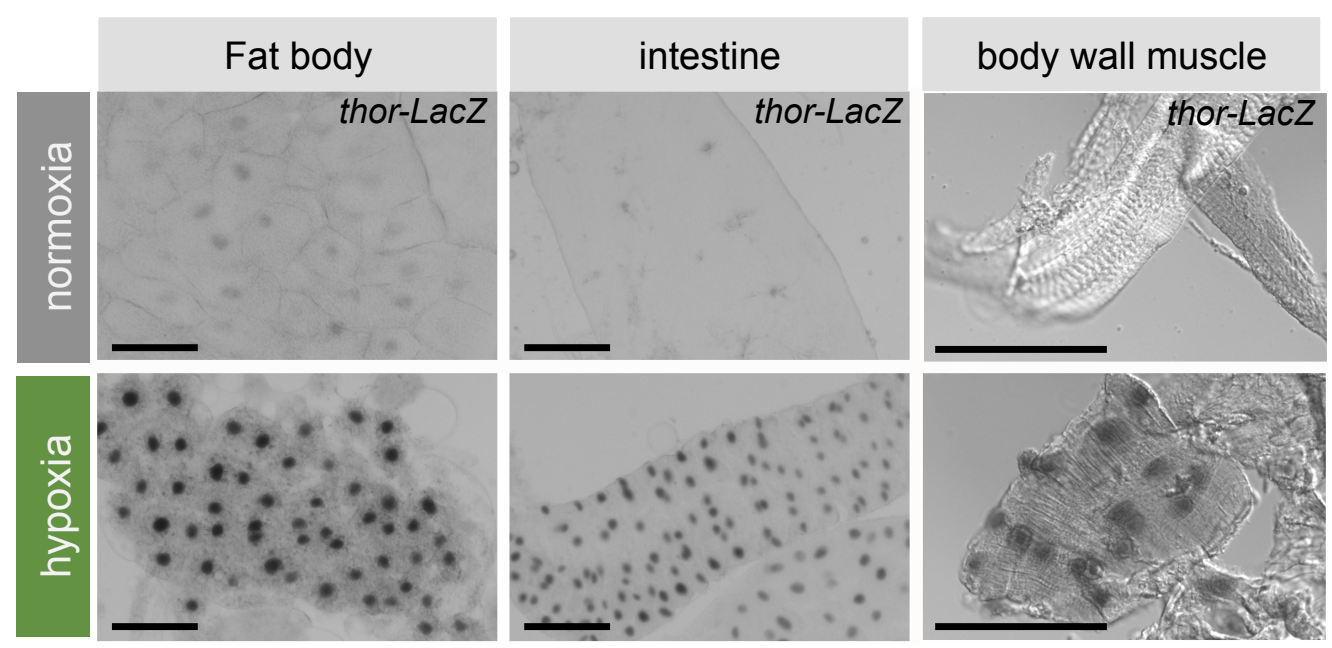

Figure 1. Hypoxia induces FOXO activity. (A) FOXO staining of 96-hour AEL $w^{1118}$ larval fat bodies following exposure to hypoxia for two hours. Nuclei are stained with Hoechst (bottom panels). Scale bar is $25 \mu \mathrm{m}$. (B) $4 e-$ $b p$ mRNA levels measured by qRT-PCR in control $\left(w^{1118}\right)$ and foxo mutant (foxo $\left.o^{494}\right)$ following B) 6 hours of $5 \%$ $\mathrm{O}_{2}$ hypoxia in larvae, C) 6 hours of $1 \% \mathrm{O}_{2}$ hypoxia in larvae, or D) 16 hours of $1 \% \mathrm{O}_{2}$ hypoxia in adults. $\mathrm{N}>6$ cohorts of animals per condition. Data represent mean + SEM. * p, 0.05, two-way ANOVA followed by post-hoc ttest. (E) LacZ staining in tissues of thor-LacZ larvae following two-hour exposure to $5 \% \mathrm{O}_{2}$. Scale bar is $100 \mu \mathrm{m}$ 

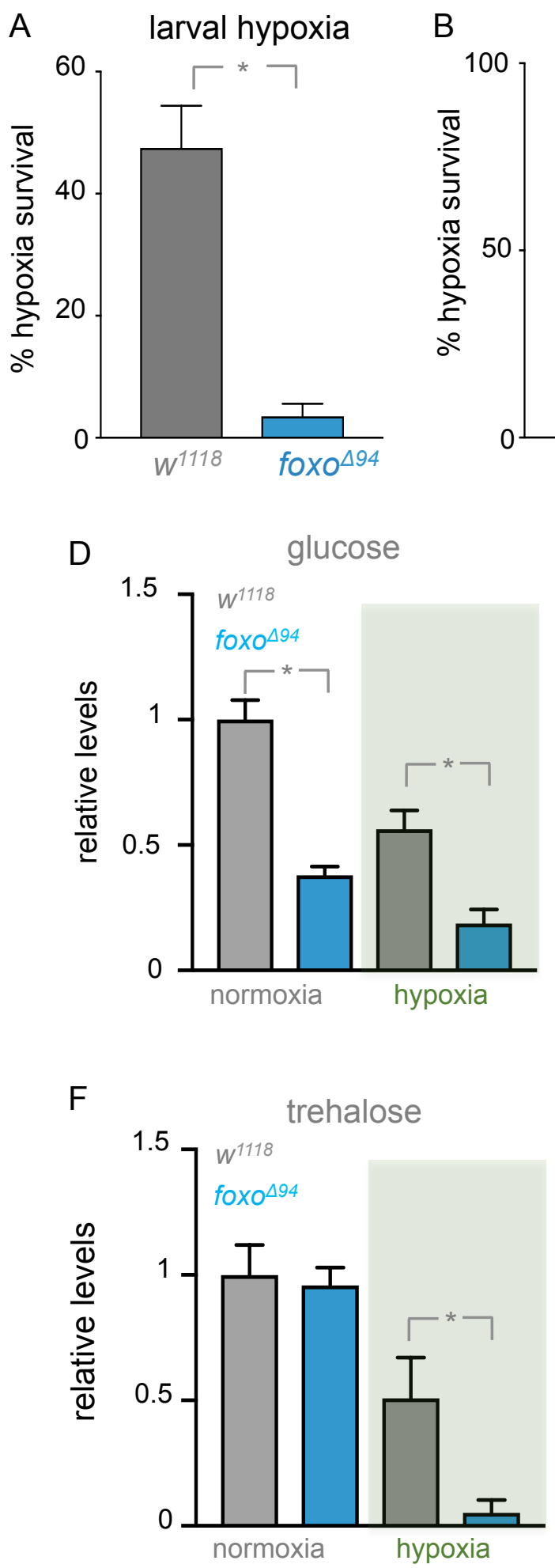
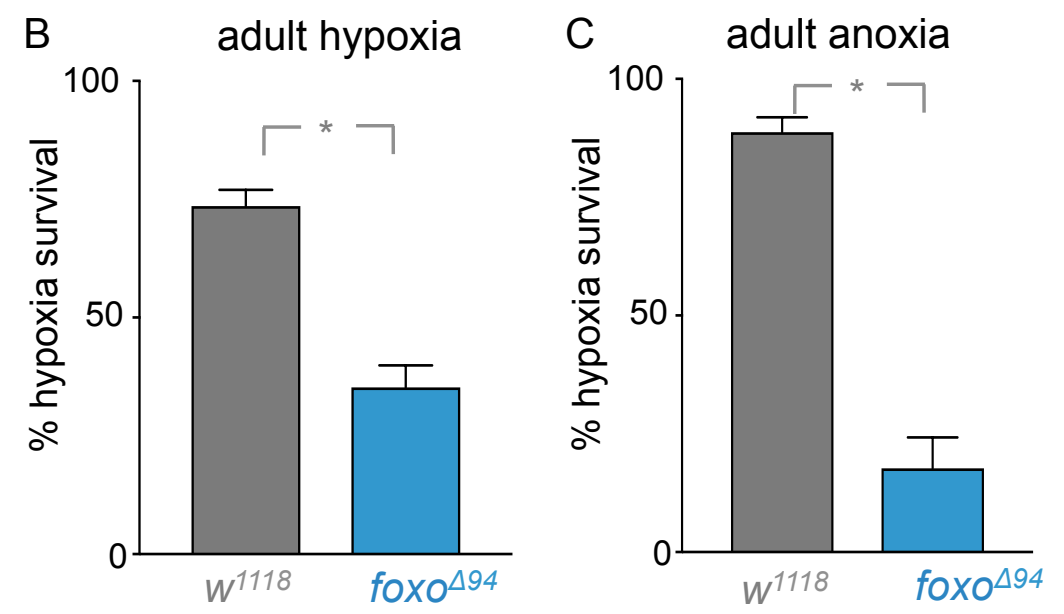

E

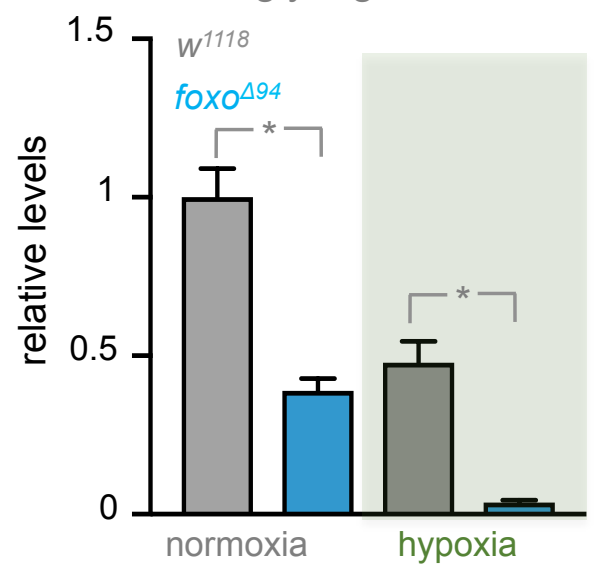

G

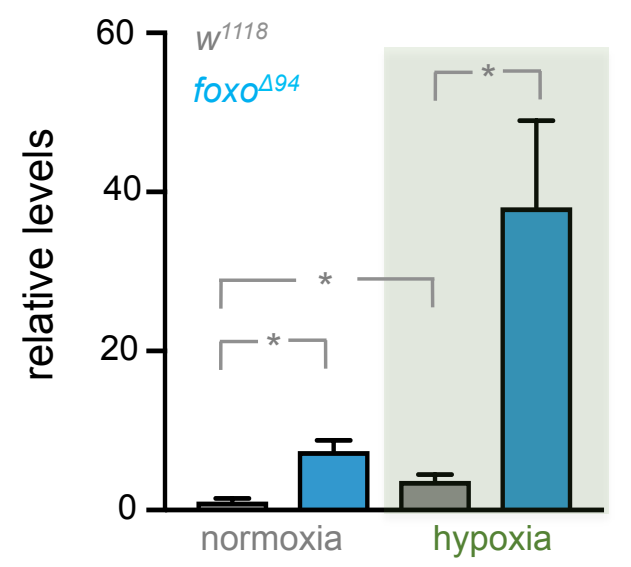

Figure 2. FOXO is required for hypoxia tolerance. (A) Control $\left(w^{1118}\right)$ and foxo mutant $\left(f o x o^{494}\right)$ animals were exposed to hypoxia $\left(5 \% \mathrm{O}_{2}\right)$ throughout their larval period, before being returned to normoxia as pupae. The percentage of flies that eclosed as viable adults were then counted. (B,C) Adult control $\left(w^{1118}\right)$ or foxo mutant $\left(f o x 0^{494}\right)$ flies were exposed to either, B) 24 hours of $1 \% \mathrm{O}_{2}$ or C) 6 hours of $0 \% \mathrm{O}_{2}$, before being returned to normoxia. The percentage of viable flies was then counted. Data represent mean + SEM. ${ }^{*} \mathrm{p}<0.05$, students t-test. $\mathrm{N}>4$ cohorts of animals per condition. (D-F) Relative levels of free glucose (D), glycogen (E), or trehalose (F), in adult control $\left(w^{1118}\right)$ and foxo mutant $\left(\right.$ foxo $\left.{ }^{\Delta 94}\right)$ flies exposed to normoxia or $1 \% \mathrm{O}_{2}$ hypoxia for 16 hours. $\mathrm{n}=15$. Data represents mean $+\mathrm{SEM}$. ${ }^{*} \mathrm{p}<0.05$, students t-test. $(\mathrm{G}) L d h \mathrm{mRNA}$ levels measured by qRT-PCR in control $\left(w^{1118}\right)$ and foxo mutant $\left(\right.$ foxo $0^{494}$ ) following 16 hours of $1 \% \mathrm{O}_{2}$ hypoxia in adults. Data represent mean + SEM. * p, 0.05 , two-way ANOVA followed by post-hoc t-test. N $>10$. 
A

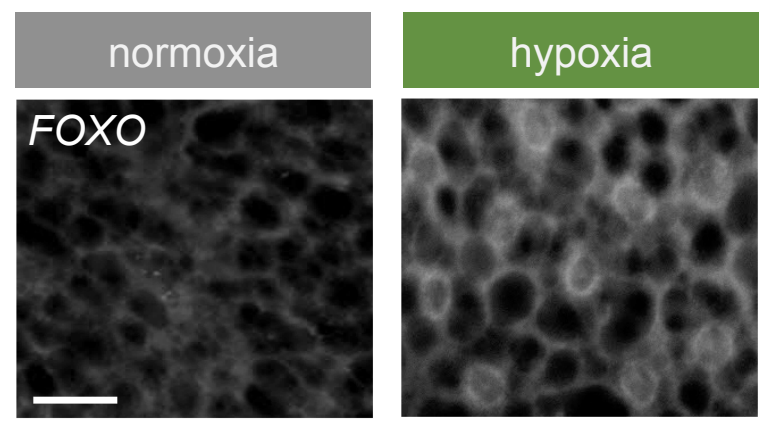

$W^{1118}$

B

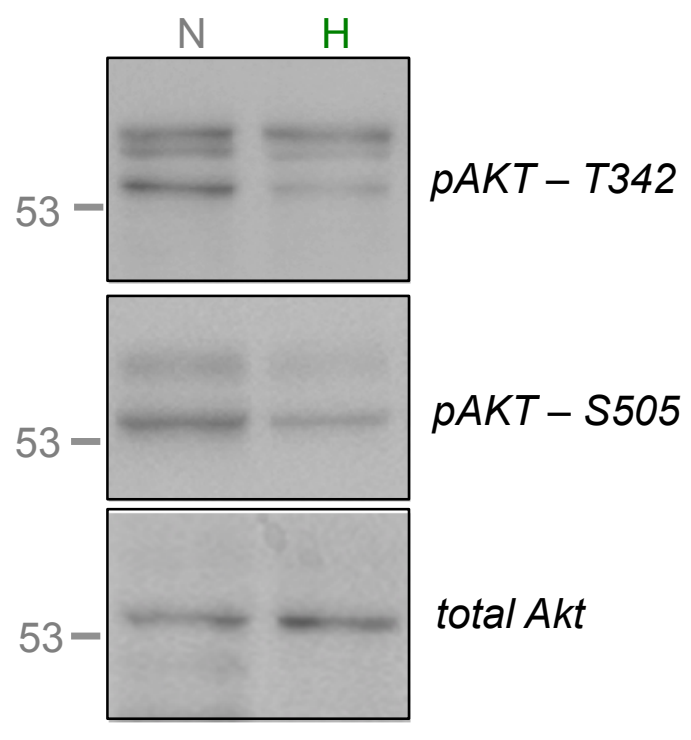

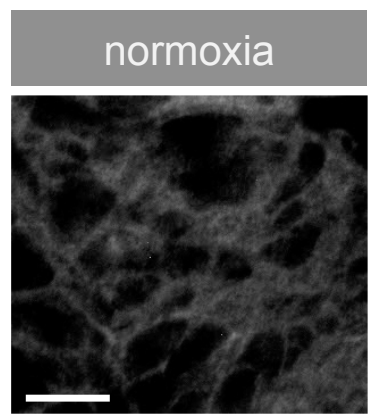

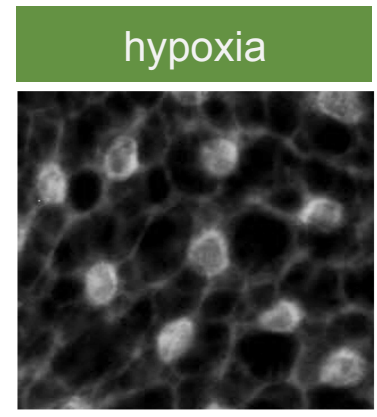

$\operatorname{sima} 07607$
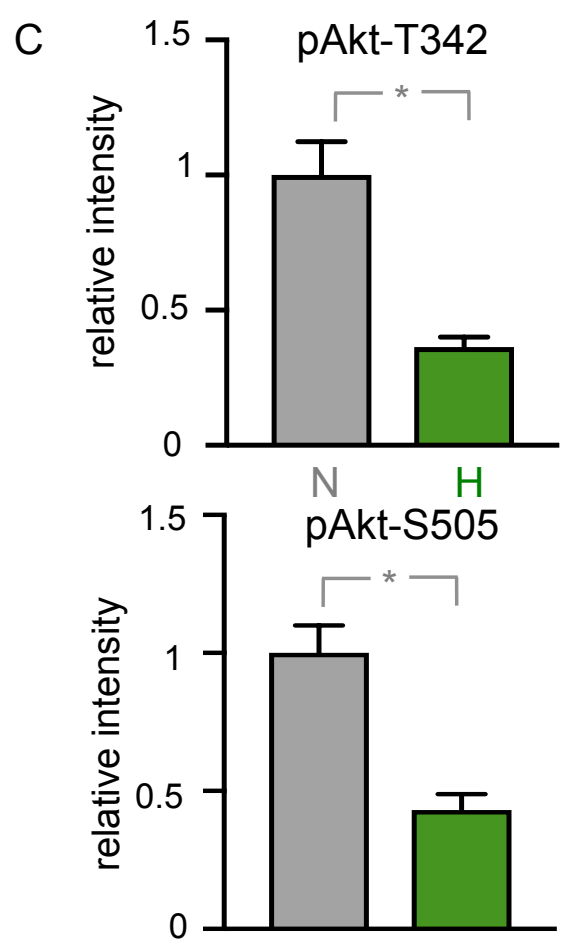

$\mathrm{N}$

$\mathrm{H}$

\section{Hypoxia (5\% oxygen)}
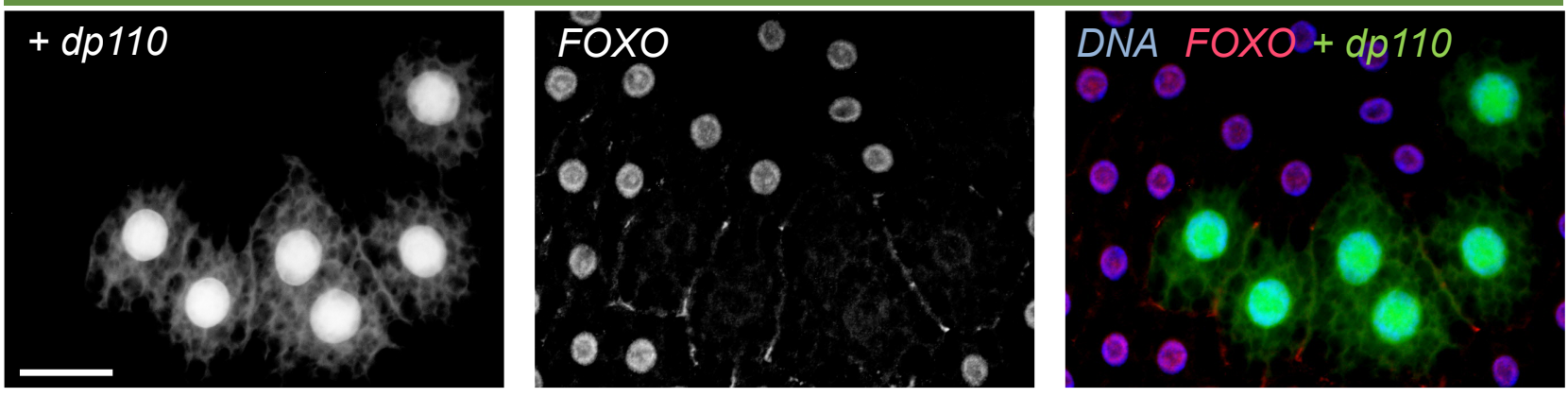

Figure 3. Hypoxia induces FOXO by inhibiting PI3K/Akt. (A) FOXO staining in fat bodies of control $\left(w^{1118}\right)$ and sima mutant $\left(\operatorname{sim} a^{07607}\right.$ ) larvae exposed to either normoxia or $5 \% \mathrm{O}_{2}$ hypoxia for 2 hours. Scale bar is $25 \mu \mathrm{m}$. (B,C) Western blot analysis of phosphorylated T342 and S505 Akt, and total Akt in control $\left(w^{1118}\right)$ larvae following 2 hours of normoxia (N) or $5 \% \mathrm{O}_{2}$ hypoxia. Quantification of blots (relative phospho-Akt intensity/total Akt intensity) is shown in (D). $\mathrm{N}=4$ per condition. ${ }^{*} \mathrm{p}<0.05$, students t-test. (D) FOXO staining in UAS- $d p 110$ overexpressing fat body clones (GFP positive). Nuclei are stained with Hoechst dye (blue). Scale bar is $50 \mu \mathrm{m}$. 

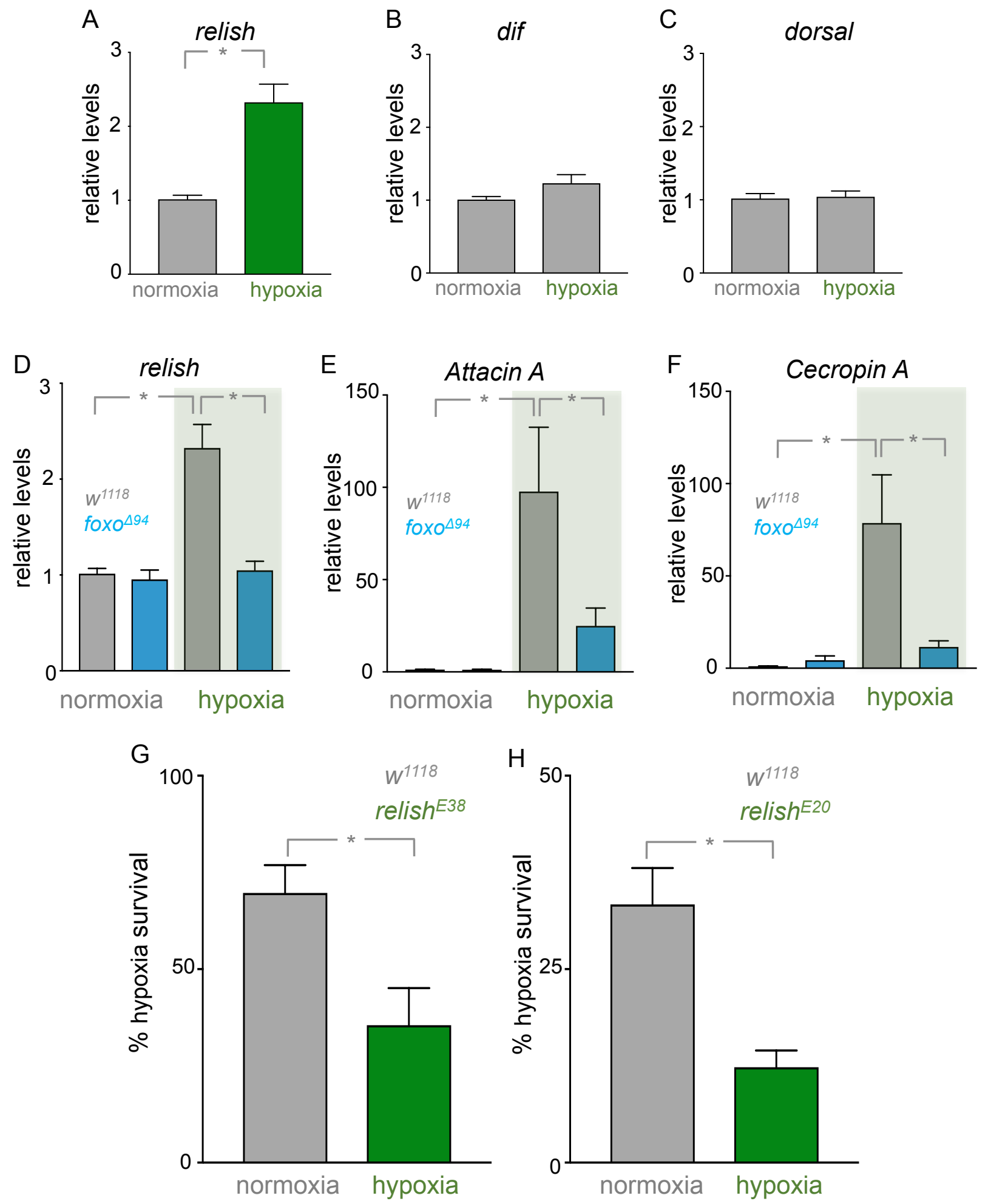

Figure 4. FOXO induces Relish-dependent hypoxia survival. (A-C) Expression levels of relish (A), dif (B), and dorsal (C) mRNA in $w^{1118}$ adult females exposed to either normoxia or 16 hours of $1 \% \mathrm{O}_{2}$. Data represent mean $+\mathrm{SEM}, \mathrm{N}=10,{ }^{*} \mathrm{p}<0.05$, students t-test. (D-F) Expression levels of relish (D), attacin A (E), and cecropin A (F) mRNA in $w^{1118}$ and foxo ${ }^{494}$ adult females exposed to either normoxia or 16 hours of $1 \% \mathrm{O}_{2}$. Data represent mean $+\mathrm{SEM}, \mathrm{N}=10,{ }^{*} \mathrm{p}<0.05$, 2-way ANOVA followed by students t-test. $(\mathrm{G}, \mathrm{H})$ Survival of adult female $w^{1118}$ or $(\mathrm{G})$ relish $^{\text {E38 }}$ or $(\mathrm{H})$ relish ${ }^{E 20}$ flies after exposure to 24 hours of $1 \% \mathrm{O}_{2}$. Data represents mean $+\mathrm{SEM}, \mathrm{N}=* \mathrm{p}<0.05$, students t-test. 

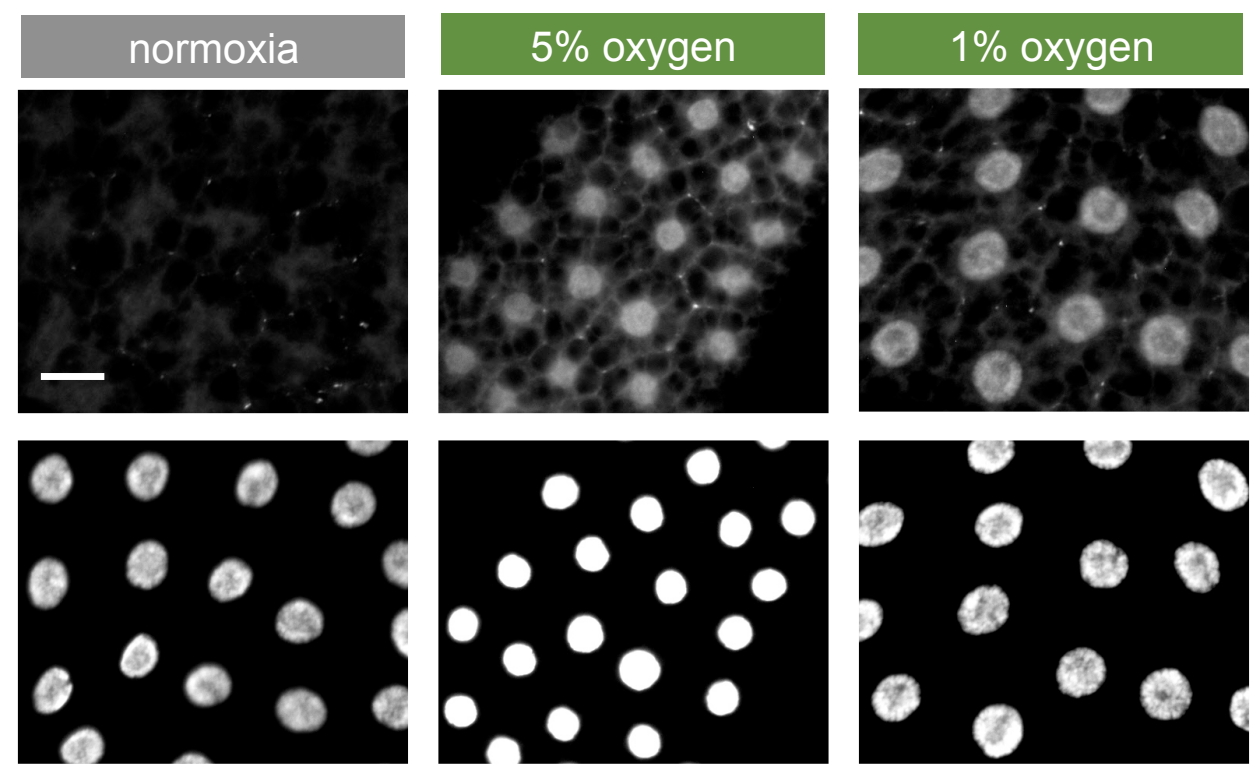

\section{larval 4E-BP $m R N A$}

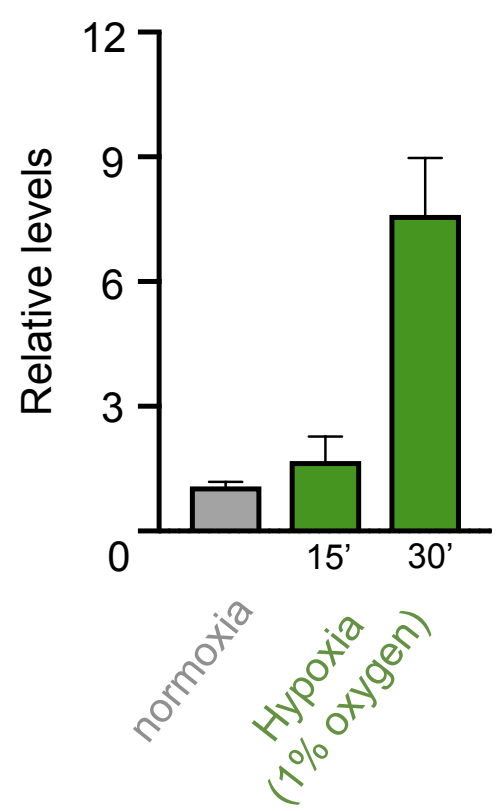

Figure S1. FOXO is induced rapidly in hypoxia. (A) FOXO staining of 96-hour AEL $w^{1118}$ larval fat bodies following exposure to hypoxia for 15 minutes. Nuclei are stained with Hoechst (bottom panels). Scale bar is $25 \mu \mathrm{m}$. (B) $4 e-b p$ mRNA levels measured by qRT-PCR in control $\left(w^{1118}\right)$ larvae exposed to either normoxia or hypoxia ( $1 \%$ oxygen) for 15 or 30 minutes. Data represent mean $+\mathrm{SEM}, \mathrm{N}=10,{ }^{*} \mathrm{p}<0.05$, students t-test. 


\section{adult starvation}

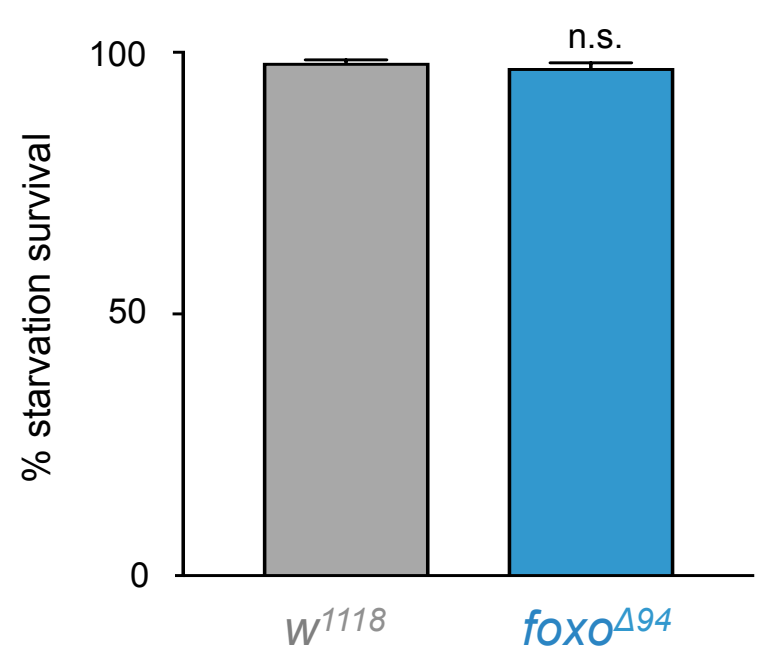

Figure S2. foxo mutant survival is not affected by short term nutrient deprivation. (A) Survival of adult female $w^{1118}$ and foxo ${ }^{494}$ flies 2 days after starvation for 24 hours. Data represented as mean + SEM for $\mathrm{n}=4$ groups of 20 flies. 
larval relish $m R N A$

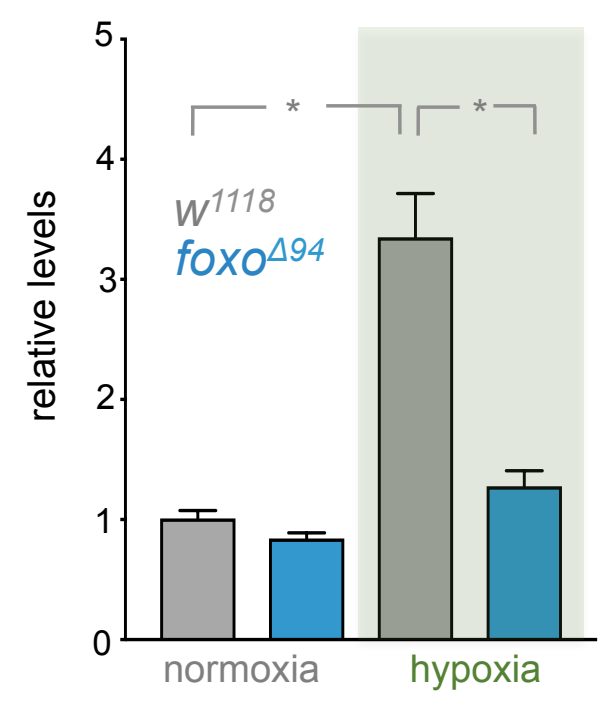

larval attacin A mRNA

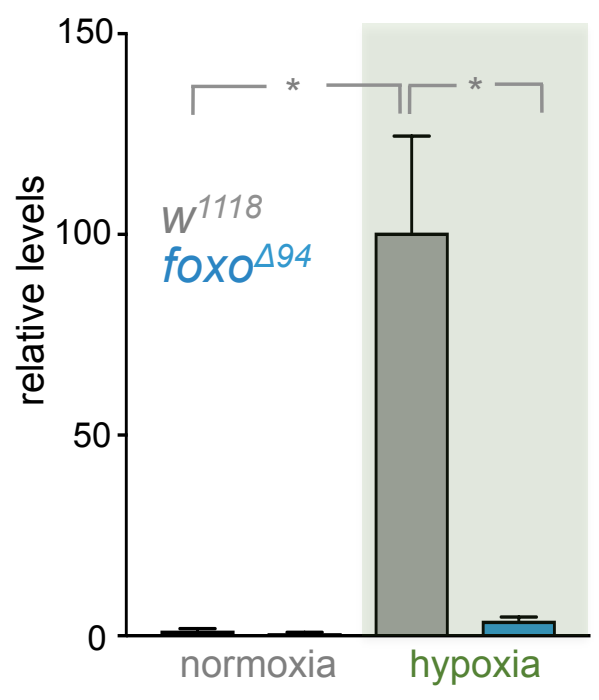

Figure S3. relish is induced by FOXO in hypoxic larvae. Expression levels of (A) relish or (B) attacin A mRNA in $w^{1118}$ and foxo ${ }^{494}$ larvae exposed to $5 \% \mathrm{O}_{2}$ for 6 hours. Data represent mean + SEM, $\mathrm{N}=10,{ }^{*} \mathrm{p}<0.05$, 2-way ANOVA followed by students t-test. 